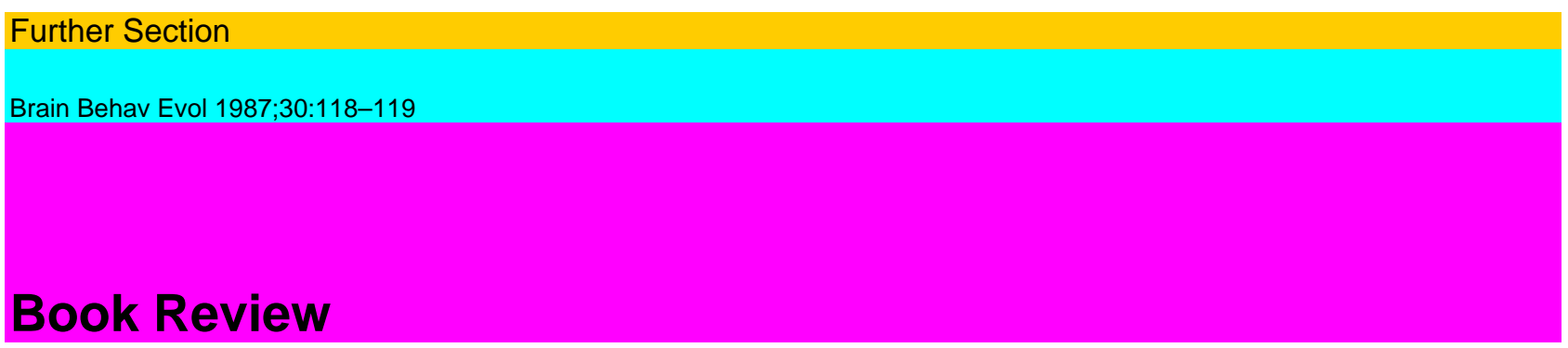

\title{
Andre Parent
}

Comparative Neurobiology of the Basal Ganglia

Wiley Series in Neurobiology Wiley, New York 1986 approx. 352 pp., approx. US\$ 80.50 ISBN 0-471-80348-0

During the last 15 years, the study of the basal ganglia has become one of the most active areas of research in the neurosciences. The use of new and powerful methods, such as immunohistochemistry for the localization of neurotransmitters and various axonal transport techniques for the tracing of neuronal connections, has led to a tremendous expansion in our knowledge of the basal ganglia. Although several excellent reSgWs of the basal ganglia in mammals have already been published, Comparative Neurobiology of the Basal Ganglia by Andre Parent is the first review that deals "Wh what is currently^known about these brain structures in both mammals and nonmammals.

After an introductory chapter that ${ }^{\wedge}$ roludes an overview of the basal ganglia and vertebrate phylogeny, the next fouff chapters give a detailed account of the anatomical and functional organization of the basal ganglia (or their probable homologues) in representatives of each of the major groups of non-mammalian vertebrates, namely fish, amphibians, reptiles, and birds?Me last five chapters are devoted to studies of basal ganglion structures in mammals. In these chapters, a detailed account is given of the trrrcajisic as well as extrinsic connections and neurotransmitter content of the various basal ganglion structures. Moreover, models are presented that summarize some of the major organizational features of the core structures of the basal ganglia.

On the whole, the main purpose of this bookMiES to review our present knowledge of the organizatimu and function of the basal ganglia in both nonmammals and mammal $\wedge$ ys well served. This book is certainly up-to-date; of the approximately 900 references, 200 are dated 1984 or more recently?! Moreover, the author has to be complimented for pro-Kiting a book, that, despite its difficult topic, is exciting to read. Especially for workers on mammalian brains who are generaflJSnot familiar with the fore-

jbfijjjfr- morphology of nonmammalian vertebrates, brief surveys of telencephalic organization $\mathrm{i}^{\wedge} \wedge$ Bie var $-\wedge$ ablasses of vertebrates are provided.

Although, in my opinion, this book is an excellent monograph on the organization and function of the basal ganglia in vertebratesjfsome criti $\wedge$ S-com-ments have to be made. In the first $\operatorname{chapt}^{\wedge} \wedge$ for $\mathrm{e}^{\wedge} \wedge \mathrm{H}$ pie, the author, dealing with the nomenclature of the basal ganglia, does not provide criteria for homolo-gizing the presumed basal ganglia stKUCtures in nonmammalian vertebrates with those in mammals. However, on reading the chapters on forebratri; organization in fishes, amphibians, $\operatorname{rej}^{\wedge}$ nes, and birds, several criteria forjagfining the basal ganglia in non-mammals gradually emerge. 
These inxlluide topographical position, connectjsths and neurotransmitter content. It would have been better, in such a monograph, to start wi^these Gmteria and determine, in the subsequent chapters, how far the probable homologous structures in the various classes of vertebrates meet these criteria. A second point of criticism concerns the comparative character of this book. In general, the author compares the presumed basal ganglia structures in each class of nonmammalian vertebrates directly with those of mammals. Comparisons between the nonmammalian brains themselves are scarce. Consequently, a cell mass, for example, that lies within the confines of the lateral forebrain bundle in reptiles (the anterior entopeduncular nucleus) is considered to be the homologue of the mam-maliairaubthalamic nucleus rather than of the mammalian entopeduncular nucleus because of its reciprocal connjgtions with the globus pallidus. In birds, a cell massraith an identical $\mathrm{po}^{\wedge} \mathrm{bn}$, but termed the anterior nucleus of the ansa lenticularis, also has reciprocal connections with the globus pallidus (paleostriatal prirmuvum) and is likewise considered as homologous to the mammalian subthalamic nucleus. However, it is left to the reader to con $<$ Wde that, in fact, the anterior entopeduncular nucleus of reptiles and the anterior nucleus of the ansa lenticularis in birds are probably homologous structures. This may seem to be a minor point, but any comparative neu-robiologist should be concerned not only with the extent to which the basal ganglia strucffies in nonmammalian brains meet the cmreria used for mamBook Review

119

malian basal ganglia, but also with how the basal ganglia may have cnanged during evolution. A third point of cnffinsm concerns the way in which the author deals with the evolution of the catecholamine $^{\wedge}{ }^{\wedge}$ gic system $J^{\wedge} \wedge$ rertebrat $^{\wedge}$ In agreement withjhis previous reviews on this topic [ParentM979; Parent et al., 1984] the author still bjgBfiyes that the trans $\wedge \wedge$ S from aquatic life to terrestrial life is marked by the appearance of midbrain dopaminergic cell groups (ventral tegmental area, substantia nigra). This belief has led to a certain degree of 'wishful thinking', namely that a cell group at the dimesencephalic transitional zone, termed the posterior tubercular nucleus, has to be considered a diencephalic cell mass in fishes, but a mesencephalic one in amphibians. However, the naked facts aregchat catechola-minergic (probably dopaminergic) cells are not present in the midbrain of bony fishes and anurans. Only in urodeles, catecholaminergic cells are found in the midbrain. Moreover, the assumption that the presence of midbrain dopaminergic cell groups coincides with the transition from aquatic to terrestriajEife, as suggested by Parent et al. [1984] is hardly tenable, as recent investigations have revealed that some cartilaginous fishes posses distinct midbrain dopaminergic cell groupsKhese results emphasize once again that we are still at the beginning of a full understanding of the evolujlon of the basal ganglion structures. Desjale these shortcomings, this monograph is a very good basis for future researjsnlon the basal gang-

lia in both mammals and nonmammals. I would recommend it to all comparative neurob ${ }^{\mathrm{TM}}$ bgists (in- $\wedge \mathrm{j} \wedge$ ing researchersmn mammalian brains). As mentioned earlier, this book is the first attempt to bring together what is currently known about the basal gangliafin ver^Srates. It has become clear that our knowledge of the basal ganglia in anamniotes $\mathrm{i}^{\wedge} \wedge \mathrm{Ssj}$ meager. However, I believe that $\mathrm{v}^{\wedge}$ miin anothe $\wedge \mathrm{O}$ years it wiUIoe possible to write a sequel to this book in which our knowledge of basal ganglion structures in anamniotes is more balanced with that in amni-otes. Credit should be given to Andre Parent for having shown us the way to do so.

Wil J.A.J. Smeets, Amsterdam 
Parent, A.: Monoaminergic systems of the brain; in Gans, Northcutt, Ulinski, Biology of ^ Reptilia, vol. 10 (Academic Press, London-New York 1979).

P. Parent
A.; Poitras
D.; Dube
L.: Comparative anatomy of central monoaminergic systems; in Bjork-lund Hokfelt
Handbook of Chemical neuroanatomy
vol. 2 (Elsevier
Amsterdam 1984). 